\title{
Prediction of PM2.5 Purification Time of Air Purifier under High Elevation Angle and High Wind Speed
}

\author{
Jin WuFeng ${ }^{1}$, Wang Cheng $^{1}$, Shi Chong ${ }^{1}$, and Wang Zhiqiang ${ }^{1 *}$ \\ ${ }^{1}$ School of Mechanical Engineering, Tianjin University of Commerce, Tianjin, China
}

\begin{abstract}
In order to achieve the best purification effect of PM2.5 at different personnel positions, it is necessary to study the PM2.5 purification time at different personnel positions in the room when the influence factors change, and establish a prediction model of the purification time.In this paper, air purifier and room models were established for simulation research. Purification time of air purifier at different locations in the room was taken as regression data, and multiple linear regression method was adopted to obtain the relationship between each impact factor and PM2.5 purification time, and a prediction model of PM2.5 purification time at different personnel locations in the room was proposed.The results of this study provide a theoretical basis for putting forward the whole intelligent scheme of air purifier.
\end{abstract}

\section{Introduction}

For fixed-position air purifiers, the control of PM2.5 concentration in the whole space has a certain limit, and in the practical application process, the single operation mode is easy to cause the indoor PM2.5 concentration is not uniform [1-3], and the reduction effect of PM2.5 in the local breathing area of different personnel positions is not sufficient.Therefore, in order to effectively solve these problems, PM2.5 purification time at different personnel locations can be taken as the basis, and each operation mode of air purifier can be reasonably combined. Finally, through intelligent control, the location in the room with longer PM2.5 purification time can be strengthened.

From the perspective of the intelligent research status of fixed air purifier, with the development of smart home, as a part of air purifier, scholars have conducted more studies on intelligent remote control, intelligent perception of indoor PM2.5 concentration to adjust the working mode of air purifier, real-time regulation and other aspects.Tang Yunfeng started with improving the control system of air purifier to improve its intelligence level. This control system can control the air purifier through mobile APP and automatically adjust the working state according to the indoor air quality [4].Han Bin proposed an air purifier with intelligent perception of indoor personnel distribution, which can move intelligently according to the density of indoor personnel distribution [5].The above research mainly based on indoor air quality to achieve intelligent automatic control of air purifier, and this paper mainly from the realization of indoor different personnel positions at the best purification time as a starting point to improve its intelligence.There are many factors that affect the purification time of air purifiers. In the actual application process, the application environment of air purifiers is not unchanged. When the initial concentration of PM2.5, room size, number of personnel, location, relative humidity, air supply speed, air supply direction and other changes will affect the purification time [6-7].In this paper, the PM2.5 purification time at different personnel locations in the room was predicted and analyzed based on various influencing factors.

When exploring the factors influencing the purification efficiency of air purifiers and the intelligent design, they are basically based on the indoor air quality and do not pay attention to the difference of the purification time at different indoor locations. There are few studies on the prediction of the purification time.And it is basically carried out in a fixed-size environmental laboratory chamber, without considering the use of air purifiers in different room sizes.In order to improve the intelligence of air purifier and its application in various environment adaptability, this research topic in view of each operating mode of air purifier and the applicable environment intelligent overall purification time prediction scheme was proposed, suitable for a variety of different location conditions room cleaning time prediction, and in this paper, the air purifier high elevation, large wind speed operation mode study, discuss the application of environment purification of indoor location different personnel time and associated role relationship between each influence factor, finally put forward the purification time prediction model, intelligent overall scheme is put forward for air purifier laid the foundation.

\section{Experimental research}

This experiment is carried out in a standard experimental chamber set according to national standards. The

\footnotetext{
* Corresponding author: zqwang@ticu.edu.cn
} 
experimental data are mainly used for the experimental verification of CFD numerical simulation, and provide data support for the subsequent establishment of mathematical prediction model.

\subsection{Experimental bench}

This experimental bench is located in a laboratory of a university in Tianjin. According to GB/T 18801-2015 Air Purifier, the standard experimental bench(indoor chamber) is set at $3 \mathrm{~m}^{*} 6.15 \mathrm{~m} * 3.2 \mathrm{~m}$, the temperature is set at $25 \pm 2{ }^{\circ} \mathrm{C}$, and the humidity is set at $50 \% \pm 10 \%$.An air purifier is placed in the corner of the cabin. The parameters of the equipment and test instruments used in the experiment are shown in Table 1.

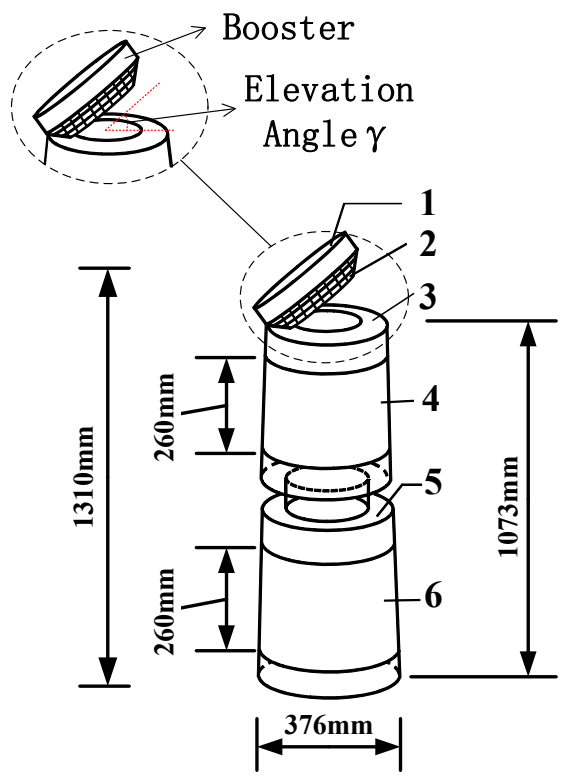

1-Inlet A1；2-Outlet B1；3-Inlet A2；4-Outlet B2； 5-Inlet A3；6Outlet B3

Fig. 1. Schematic diagram of air purifier structure.

Table 1. Instrument parameters of experimental equipment

\begin{tabular}{|c|c|c|}
\hline Name & Model & Parameters \\
\hline $\begin{array}{c}\text { Particle } \\
\text { counter }\end{array}$ & $\begin{array}{c}\text { LIGHTHOUS } \\
\text { E 3016 }\end{array}$ & $\begin{array}{c}\text { Particle size range 0.30- } \\
10 \mu \mathrm{m} \text { 。 }\end{array}$ \\
\hline $\begin{array}{c}\text { Handheld } \\
\text { anemometer }\end{array}$ & & $\begin{array}{c}\text { Measurement range is } \\
0.3 \mathrm{~m} / \mathrm{s}-45 \mathrm{~m} / \mathrm{s}, \\
\text { resolution is } 0.001 \mathrm{~m} / \mathrm{s} .\end{array}$ \\
\hline $\begin{array}{c}\text { Circulating } \\
\text { fan }\end{array}$ & & Diameter $20 \mathrm{~cm}$ \\
\hline Mixing fan & & Diameter $1 \mathrm{~m}$. \\
\hline
\end{tabular}

The air purifier used in the experiment is LG AS95GDWP2, with a CADR particulate matter value of 750 and a purification energy efficiency of 8.3. Its structure is shown in Fig.1.The air purifier is a filter-type purifier with three air supply inlets and three air return outlets. The dirty air was purified into the purifier through the return air outlets B2 and B3, and then sent into the room through the air supply outlets A2 and A3, respectively.Air purifier booster is composed of air inlet $\mathrm{A} 1$, return air outlet $\mathrm{B} 1$ and booster fan. The boost fan inhales the clean air from inlet A2 through outlet B1 and sends it into the room through the air supply inlet A1.
The Angle between the booster and the horizontal direction is the elevation Angle of the air supply $\gamma$.

The wind speeds of A2 and A3 of the air purifier inlet are $3.5 \mathrm{~m} / \mathrm{s}$ and $3.85 \mathrm{~m} / \mathrm{s}$, respectively. The wind speeds of inlet A1 are $100 \%$ ( $3 \mathrm{~m} / \mathrm{s}$ ), $50 \%$ and $25 \%$, and the air supply elevation angles $\gamma$ are $0^{\circ}, 35^{\circ}$ and $55^{\circ}$. The booster can swing in the horizontal direction of $70^{\circ}$ to supply air.

\subsubsection{Room measurement points are arranged}

According to the construction of indoor particulate matter (PM2.5) pollution control technology regulations (draft) determination of indoor PM2.5 concentrations in relevant provision, the central and cabin room corner of PM2.5 concentration measurement, and according to the diagonal decorate, this study mainly aims at the purification of PM2.5 in the office, so will arrangement of measuring points in the height of $1.1 \mathrm{~m}$ (staff sitting height), $3 \mathrm{~m}$ and $6 \mathrm{~m}$ distance air purifier, blowing direction along the diagonal direction, as shown in Fig. 2.

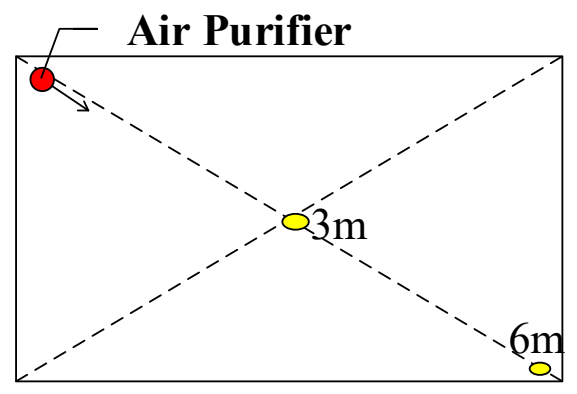

Fig. 2. Distribution of experimental measurement points.

\subsection{Experimental methods}

After the temperature and humidity of the experimental chamber are stable, the purifier should be opened to purify the room in advance, and the air purifier should be closed after ensuring that PM2.5 is less than $15 \mu \mathrm{g} / \mathrm{m}^{3}$.After that, 4 cigarettes were lit and the smoke was sent into the laboratory chamber through the connecting pipe of the particulate matter generator. After the cigarettes were burned out, the circulating fan was turned on for $10 \mathrm{~min}$ to ensure the uniform distribution of indoor PM2.5. At this time, the indoor PM2.5 concentration could reach $350 \mu \mathrm{g} / \mathrm{m}^{3}$. The purifier supplies air along the diagonal direction of the room. Each air inlet maintains the maximum wind speed and the purification time continues for $1 \mathrm{~h}$ to ensure that the concentration of PM2.5 at each measurement point in the room can be reduced to $15 \mu \mathrm{g} / \mathrm{m}^{3}$.In order to ensure the accuracy of the experiment, the average value of three measurements was adopted in the experiment.

\section{Simulation research}

\subsection{Determine the influence factor and its value range}




\subsubsection{Determination of influence factors}

There are many factors affecting the purifying effect of air purifier, mainly from the indoor environment, indoor air distribution two aspects.Based on the relative position of personnel and air purifier, the final influencing factors were determined as the initial concentration of PM2.5 $\rho$, room area $\eta$, room aspect ratio $\Gamma$, horizontal air supply Angle $\alpha$, personnel distance $\mathrm{L}$ and Angle $\beta$ between personnel and air supply direction. The relative positions of horizontal air supply Angle $\alpha$, personnel distance L and Angle $\beta$ between personnel and air supply direction of the air purifier are shown in Fig. 3.

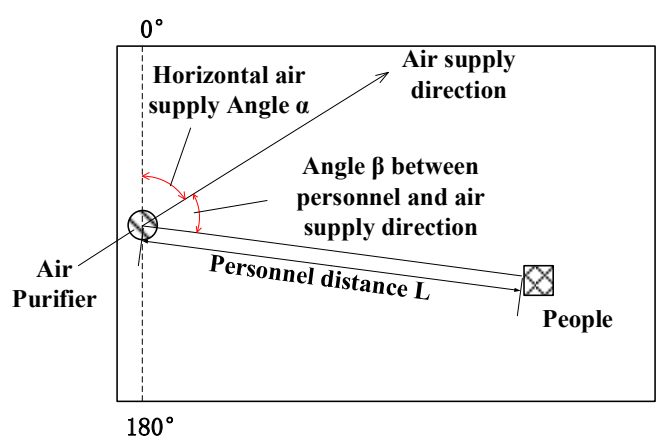

Fig. 3. Diagram of horizontal air supply Angle and relative position of personnel

\subsubsection{The value range of the influence factor}

In order to determine the simulation conditions, the range and value of influence factors should be determined according to the actual situation and research. The range of initial concentration $\rho$ of PM2.5 was 50$150 \mu \mathrm{g} / \mathrm{m} 3$, and the values were 50,100 and $150 \mu \mathrm{g} / \mathrm{m}^{3}$, respectively.The room area $\eta$ ranges from $54-90 \mathrm{~m}^{2}$, with values of $54 \mathrm{~m}^{2}, 72 \mathrm{~m}^{2}$ and $90 \mathrm{~m}^{2}$ respectively.Room aspect ratio $\Gamma$ ranges from 1.5 to 2.5 .Values are $1.5,2$ and 2.5 , respectively.The value range of horizontal air supply Angle $\alpha$ is $0-90^{\circ}$, and the value is $0^{\circ}, 45^{\circ}$ and $90^{\circ}$ respectively.The variation range of personnel distance $\mathrm{L}$ is $0-13.5 \mathrm{~m}$, and the value is $0.5 \mathrm{~m}$ each. The Angle $\beta$ between personnel and the direction of air supply varies from $0{ }^{\circ}$ to $180^{\circ}$, taking value every $30^{\circ}$.

\subsection{Model establishment}

\subsubsection{The physical model}

In the simulation process, physical models were established according to full-scale experimental models, including room and air purifier models. The boundary conditions and initial conditions are set according to the experimental data.The arrangement of measuring points is shown in Fig. 3.

The room was divided into unstructured grids. The maximum size of the grids was set as $10 \mathrm{~mm}$ and the grid quality was $0.3-0.99$. The grid quality was high and could be used for calculation.

\subsubsection{Model setting}

In addition to the mass conservation equation, momentum conservation equation and mass conservation equation, the non-reactive component transport model is also adopted in the model, and the turbulence model is selected to describe the indoor flow field.In order to simplify the calculation process, reasonable assumptions are made for the established model:

(1) Indoor air is low-speed flow and can be regarded as incompressible fluid;

(2) Conformed to Boussinesq hypothesis.

\subsubsection{Model validation}

In order to verify the accuracy of the model, the error analysis of experimental data and simulated data is shown in Table 2.It can be seen that the maximum error between the experimental value and the simulated value at each measurement point is less than $10 \%$, and the average error is about $6 \%$. It is considered that the established model is accurate and can be extended to study the model.

Table 2. Error analysis

\begin{tabular}{|c|c|c|c|c|}
\hline \multirow{2}{*}{ Point } & $\begin{array}{c}\text { Error } \\
\text { analysis }\end{array}$ & $\begin{array}{c}\text { Experimental } \\
\text { value }\end{array}$ & $\begin{array}{c}\text { Simulation } \\
\text { value }\end{array}$ & Error \\
\hline \multirow{3}{*}{$3 \mathrm{~m}$} & Max error & $75.8 \mu \mathrm{g} / \mathrm{m}^{3}$ & $69 \mu \mathrm{g} / \mathrm{m}^{3}$ & $9.86 \%$ \\
\cline { 2 - 5 } & $\begin{array}{c}\text { Average } \\
\text { error }\end{array}$ & \multicolumn{3}{|c|}{$5.97 \%$} \\
\hline \multirow{2}{*}{$6 \mathrm{~m}$} & Max error & $59.46 \mu \mathrm{g} / \mathrm{m}^{3}$ & $54.3 \mu \mathrm{g} / \mathrm{m}^{3}$ & $9.51 \%$ \\
\cline { 2 - 5 } & $\begin{array}{c}\text { Average } \\
\text { error }\end{array}$ & \multicolumn{3}{|c}{$5.39 \%$} \\
\hline
\end{tabular}

\subsection{Model extension study}

According to the layout of the experimental bench in the verification experiment and the applicable area of the air purifier, physical models with different room areas, length-to-width ratios and floor heights of $3 \mathrm{~m}$ were established. The purifier model was established in accordance with the purifier used in the experiment and set in the middle of the side wall of the room.

\subsubsection{Simulate the arrangement of measuring points}

The arrangement of measuring points in the simulated working condition followed the principle of the arrangement of measuring points in the verification experiment, and the measuring points were all arranged at the height of the $1.1 \mathrm{~m}$ breathing zone when the personnel sat down. The distance between the nearest measuring point and the air purifier is $0.5 \mathrm{~m}$, and it is evenly arranged every $30^{\circ}$ between $0^{\circ}$ and $180^{\circ}$ around the air purifier, and the distance between the other measuring points is $1 \mathrm{~m}$. The room size changes according to the aspect ratio in the working condition setting. The specific arrangement of measuring points is shown in Fig. 4. 


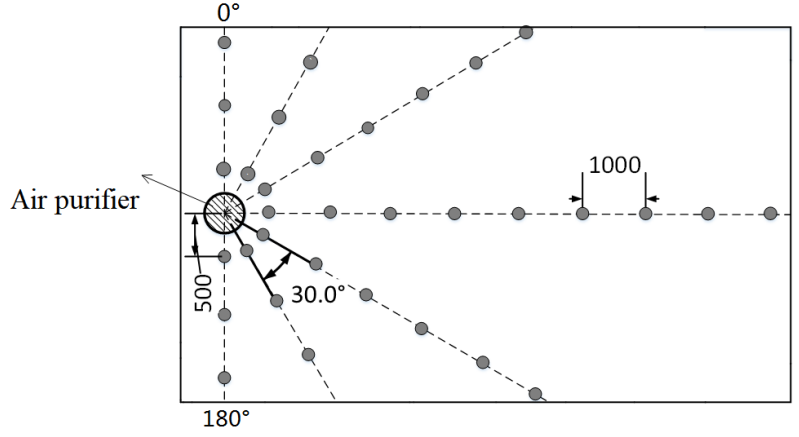

Fig. 4 Arrangement of simulated measuring points

\subsubsection{Setting of simulated working conditions}

According to the variation range influence factors and simulation values, the experimental orthogonal method was used to obtain the simulated conditions, and the Settings were shown in Table 3.

Table 3. Simulation condition table

\begin{tabular}{|l|c|c|c|c|c|}
\hline $\begin{array}{c}\text { Simulation } \\
\text { conditions }\end{array}$ & $\begin{array}{c}\boldsymbol{\eta} \\
\left(\mathbf{m}^{\mathbf{2}} \mathbf{)}\right.\end{array}$ & $\Gamma$ & $\begin{array}{c}\boldsymbol{\rho} \\
\left(\boldsymbol{\mu} \mathbf{g} / \mathbf{m}^{\mathbf{3}}\right)\end{array}$ & $\begin{array}{c}\mathbf{a} \\
\left.\mathbf{}^{\circ} \mathbf{~}\right)\end{array}$ & $\begin{array}{c}\text { Number of } \\
\text { measuring } \\
\text { points }\end{array}$ \\
\hline condition1 & 54 & 1.5 & 50 & 0 & 33 \\
\hline condition2 & 72 & 2 & 100 & 0 & 36 \\
\hline condition3 & 90 & 2.5 & 150 & 0 & 39 \\
\hline condition4 & 54 & 2 & 150 & 45 & 32 \\
\hline condition5 & 72 & 2.5 & 50 & 45 & 35 \\
\hline condition6 & 90 & 1.5 & 100 & 45 & 44 \\
\hline condition7 & 54 & 2.5 & 100 & 90 & 32 \\
\hline condition8 & 72 & 1.5 & 150 & 90 & 38 \\
\hline condition9 & 90 & 2 & 50 & 90 & 41 \\
\hline
\end{tabular}

\section{Time prediction model establishment}

\subsection{Regression data establishment}

According to the different room area and room lengthwidth ratio, the number of measuring points under each working condition is different, that is, the number of simulated data under each working condition is different. The number of measuring points under each working condition is shown in Table 3. Through simulation study, a total of 330 data were obtained when PM2.5 concentration reached $15 \mu \mathrm{g} / \mathrm{m}^{3}$ at each measurement point under conditions $1-9$, and regression data required for regression analysis were established on the basis of the data.

\subsection{Regression analysis of simulated data}

The regression data were analyzed numerically by SPSS software.In order to check whether the selected sample data meet the conditions of principal component analysis, $\mathrm{KMO}$ and Bartlett tests were first carried out on the simulated data. The results showed that the KMO value was 0.512 , greater than 0.5 , which could be used for principal component analysis. The approximate value of
Bartlett test is $134.116, \mathrm{P}<0.01$, showing significant level, and there is correlation among the variables.

By observing the scatter diagram of the simulated data, it can be concluded that there is a linear relationship between each impact factor and PM2.5 purification time. The principal components were extracted by using SPSS software for principal component analysis, and then multiple linear regression was conducted on the statistical data.

The overall significance test value of the regression equation for the prediction model of purification time is $<.0001$, and the regression equation is significant as a whole. The determination coefficient of goodness of fit of the equation was $\mathrm{R}$-square $=0.926$, and the determination coefficient of goodness of fit correction was Adj r-sq $=0.925$, indicating a high degree of fit. The significance test values of each variable were far less than 0.05 , and the regression coefficients were significant.

Through the above analysis, the relation between PM2.5 purification time and each influencing factor can be obtained under the air supply elevation Angle of $55^{\circ}$ and air supply speed of $100 \%(3 \mathrm{~m} / \mathrm{s})$ of the air purifier, as shown in Equation (1):

$$
t=-0.012 \alpha+0.379 \eta-2.030 \Gamma+0.163 \rho+0.002 \beta+0.052 L-6.957
$$

According to Equation (1), the purifying time of the air purifier is positively proportional to the room area, the initial concentration of PM2.5, the distance between personnel and the Angle between personnel and the air supply direction, and inversely proportional to the horizontal air supply Angle and the ratio of room length to width $\Gamma$.

\subsection{Accuracy test of prediction model}

The simulated working conditions 2 and 3 were selected to verify Equation (1), and the predicted value was compared with the simulated value, as shown in Fig. 5. It can be seen from the figure that the predicted value and the simulated value have the same change trend.In Equation (1), there is a linear relationship between each impact factor and PM2.5 purification time, while there is an error between the simulated value and the predicted value.The maximum error between the predicted value and the simulated value in condition 2 is $5.09 \%$, and the average error is $3.28 \%$. The maximum error between the predicted value and the simulated value of working condition 3 is $3.58 \%$, and the average error is $1.95 \%$. The maximum error of the predicted and simulated values of the PM2.5 purification time prediction model is less than $6 \%$, and the average error is less than $4 \%$. Therefore, it can be considered that the PM2.5 purification time prediction model of regression analysis is accurate. 


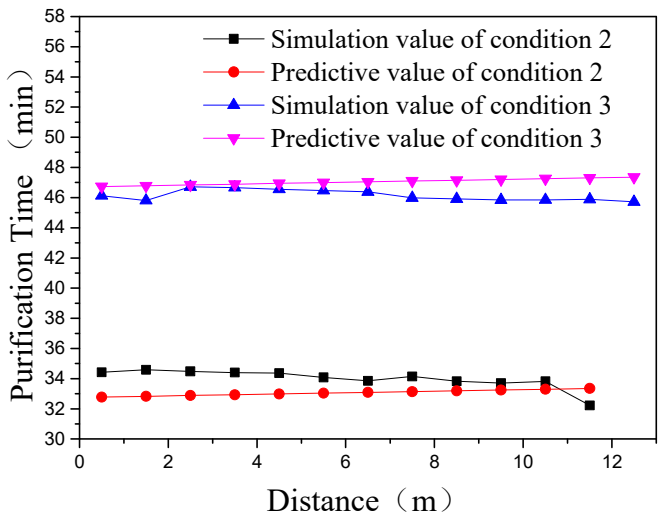

Fig. 5. Comparison of predicted values and simulated values of the prediction model

\subsection{Contribution rate analysis of each impact factor}

The absolute value of the standardization coefficient directly reflects the influence degree of the independent variable on the dependent variable, that is, the contribution rate of the impact factor.According to the regression analysis, each impact factor has a significant correlation with the PM2.5 purification time. According to the standardization coefficient in Table 7, the contribution rate of each impact factor can be obtained, as shown in Fig.6.

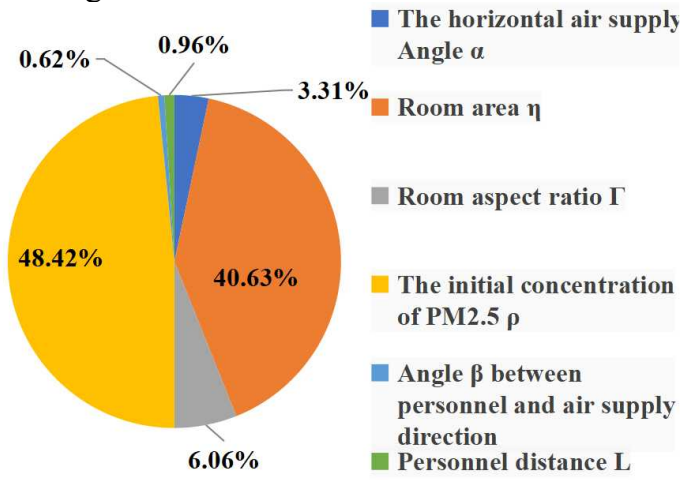

Fig. 6. Contribution rate of impact factors

Under this operation mode, the initial concentration of PM2.5 $\rho$ of the room has the greatest effect on the purifier purification time, and the contribution rate of the influence factor is $48.42 \%$. The room area $\eta$ had the second influence on the purifier purification time, and the contribution rate of influencing factors was $40.63 \%$. The sum of the contribution rate of the initial concentration of PM2.5 in the room $\rho$ and the area of the room on the purifier purification time reached $89.05 \%$, indicating that the influence of the initial concentration of PM2.5 in the room and the area of the room on the purifier purification time was extremely significant. In the practical application process, attention should be paid to the influence of the initial concentration of PM2.5 and the area of the room. The sum of contribution rates of horizontal blowing Angle, room aspect ratio and personnel position to purifier purification time is about
$10 \%$, and the contribution rates of personnel position to purifier purification time are all less than $1 \%$.

Under high elevation Angle and high wind speed of air purifier, the contribution rate of personnel position (the Angle between personnel and air supply direction and personnel distance) to PM2.5 purification time is very small. Under this operation mode, PM2.5 purification time at different positions in the room is relatively uniform. This is because PM2.5 is small particulate matter, easy to flow with the indoor air flow, while the operation mode of high elevation Angle and high wind speed has a larger disturbance range of air flow in the room, and the air flow speeds up, so that the difference in PM2.5 purification time at different locations in the room is small.Therefore, when proposing the overall operation scheme of the air purifier, the uniform purification time at different personnel positions under this operation mode can be considered.

\section{Conclusion}

By means of simulation and regression statistics, the purification time prediction of the purifier is studied and the following conclusions are drawn.

(1) Using SPSS software to conduct regression statistical analysis on the simulated data, it is concluded that there is a linear relationship between the influence factors and the purifier purification time; The purifying time of the air purifier is directly proportional to the room area, the initial concentration of PM2.5, the distance between the personnel and the personnel Angle, and inversely proportional to the blowing Angle and the room length-to-width ratio.

(2) Based on the contribution rate analysis of impact factors, it can be concluded that the concentration of PM2.5 in a room and the area of the room have a significant impact on the purification time of the air purifier. When choosing the operation mode of the air purifier, the influence of the area of the room and the initial concentration of PM2.5 in the room should be taken into account.

(3) Because the contribution rate of impact factor of personnel location in this operation mode is very small, the purification time of each personnel location can be very uniform; When there are many people in the room and the location is relatively scattered, this operation mode can also be considered to make the PM2.5 purification time of each person in the room uniform and consistent.

The research results of this paper can lay a foundation for the establishment of the whole scheme of intelligent purification time prediction.

\section{References}

1. Hyungyu Park, Seonghyun Park, Janghoo Seo. Evaluation on Air Purifier's Performance in Reducing the Concentration of Fine Particulate Matter for Occupants according to its Operation Methods. 2020, 17(15) 
2. Li Xiyu, Liu Weilong.Numerical Simulation Study on Airflow Distribution of Indoor Air Purifier [J]. Home Appliance Science and Technology, 2013(05): 86-88.

3. Han Xingxing, Liu Xin, Huo Xingkai, Xie Junlong.Analysis of purifying strategy and influencing factors of air-conditioned room in summer [J]. Fluid Machinery,2018,46(02):84-88.

4. Tang Yunfeng. Design and Implementation of Intelligent System for Air Purifier [D]. Southeast University, 2015.

5. Bin Han. An air purifier control system based on personnel distribution.

6. Elizabeth Cooper, Yan Wang, Samuel Stamp, Esfandiar Burman, Dejan Mumovic,Use of portable air purifiers in homes: Operating behaviour, effect on indoor PM2.5 and perceived indoor air quality,Building and Environment, Volume 191, 2021, 107621, ISSN 0360-1323

7. Li Qing, Shen Henggen, Yang Xuebin, Cui Jingjing.Influence of air purifier placement position on flow field and PM2.5 purification effect [J]. Building Thermal Energy Ventilation and Air Conditioning,2017,36(04):35-38+43. 